\title{
UV-Triggered Polydopamine Secondary Modification: Fast Deposition and Removal of Metal Nanoparticles
}

\author{
Yi Zeng, Xin Du,* Wei Hou, Xiaojiang Liu, Cun Zhu, Bingbing Gao, Liangdong Sun, \\ Qiwei Li, Junlong Liao, Pavel A. Levkin,* and Zhongze Gu*
}

\begin{abstract}
Since the first report in 2007, polydopamine (PDA) coating has shown great potential as a general and versatile method to create functional nanocoatings on arbitrary substrates. Slow kinetics and poor controllability of the coating and secondary modification processes, however, have limited the further development of this attractive method. In this work, it is demonstrated that UV irradiation at $365 \mathrm{~nm}$ significantly accelerates the process of secondary modification of a PDA-coated surface. The kinetics of both thiol and amine modifications of PDA are increased 12-fold via UV irradiation, while the kinetics of metal ion reduction at the PDA interface is increased more than 550 times. Moreover, it is demonstrated that irradiating a PDA/metal nanoparticle composite surface with UV light at $254 \mathrm{~nm}$ leads to dissolution of the deposited metal nanoparticles (MNPs). Finally, grayscale metallic patterns, dynamic deposition, and removal of MNPs on PDA surface are realized with the proposed method.
\end{abstract}

\section{Introduction}

A decade has passed since the discovery of polydopamine (PDA) coatings. ${ }^{[1]}$ This unique material, inspired by the versatile adhesive property of mussel foot, has drawn tremendous attention from both scientific research and industry fields. ${ }^{[2,3]}$ PDA reveals a material-independent adhesion property, as well as reactivity to thiols, amines, and metal ions $\left(\mathrm{Ag}^{+}, \mathrm{Au}^{3+}\right.$, and $\left.\mathrm{Cu}^{2+}\right),[1,4,5]$

Y. Zeng, Prof. X. Du, W. Hou, X. Liu, Dr. C. Zhu, L. Sun, Q. Li, J. Liao, Prof. Z. Gu

State Key Laboratory of Bioelectronics

School of Biological Science and Medical Engineering

Southeast University

Nanjing 210096, China

E-mail:du.xin@seu.edu.cn; gu@seu.edu.cn

Prof. X. Du, Dr. P. A. Levkin

Institute of Toxicology and Genetics

Karlsruhe Institute of Technology (KIT)

76344 Eggenstein-Leopoldshafen, Germany

E-mail: levkin@kit.edu

Dr. B. Gao

School of Pharmaceutical Sciences and School of Biotechnology

and Pharmaceutical Engineering

Nanjing Tech University

Nanjing 211816, China allowing the secondary modification of these coatings with organic chains or metal nanoparticles (MNPs). Combining the two properties above, PDA coatings open up a new horizon to "functionalize almost any surface on demand," thus fulfilling requirements from fields as varied as material science, chemistry, and biomedical science. Therefore, over the past decade, PDA coatings have been widely used to construct functional surfaces with biocompatibility, ${ }^{[6,7]}$ conductivity, ${ }^{[8,9]}$ antibacterial/ antifouling properties ${ }^{[10-12]}$ and superhydrophobicity, ${ }^{[13]}$ on various substrates and interfaces. PDA coating has also been employed in drug delivery, wound healing, thermal therapy, organic catalysis, and so on, due to its properties such as nontoxicity, self-healing, NIR responsibility, and electron transfer property. ${ }^{[3,14-17]}$

The main drawbacks of state-of-the-art PDA coatings lie in the low efficiency and poor controllability of PDA-coating and secondary-modification processes. ${ }^{[18-20]}$ In a typical PDA-based surface functionalization process, the dopamine polymerization (PDA coating) process takes $\approx 24 \mathrm{~h}$, and the secondary modification process takes another 3-48 $\mathrm{h}$ (depending on modification reagents). Both processes are not just time-consuming, but also spatially and temporally uncontrollable, causing technical limitations in industrial applications and difficulty in programming the surface characteristics precisely. ${ }^{[18,21]}$ During the past decade, great efforts have been made to improve the efficiency and controllability of PDA coating method. ${ }^{[12,22-25]}$ Currently, by microwave assistance or adding specific oxidants, PDA coatings with improved uniformity and stability could be obtained in hours or even in $15 \mathrm{~min},{ }^{[12,20]}$ and spatiotemporal control of the coating process could be realized through UV light irradiation. ${ }^{19,26]}$

However, the secondary modification of PDA coatings remains the "Achilles' heel." To introduce more functions onto PDA coatings, the substrate must be immersed in modification solution for a long time (typically $3-12 \mathrm{~h}$ in thiol or amine solution and $6-48 \mathrm{~h}$ in metal salt solution $\left.{ }^{[1]}\right)$. Moreover, no method has been reported to effectively control the degree and location of the secondary modification on PDA coatings, making it difficult or even impossible to precisely program the surface properties. Thus, to further develop PDA coatings, it remains a major challenge to develop facile strategies for the rapid and controllable secondary modification of PDA coatings. 


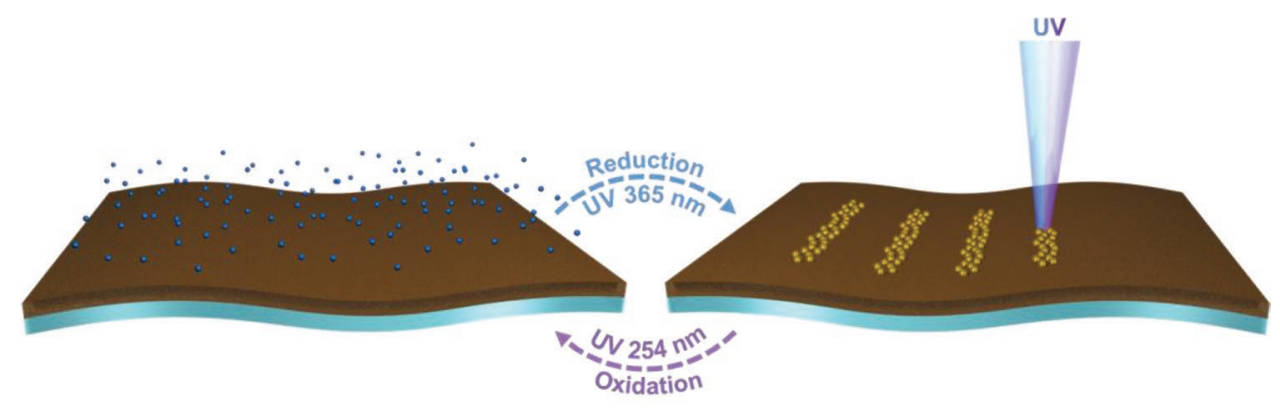

$M^{n+}$ MNPs $\square$ PDA $\square$ Substrate

Figure 1. Schematic representation of PDA-assisted deposition and removal of MNPs. Under 365 nm UV light, metal cations are rapidly reduced and high-density nonaggregated MNPs are in situ deposited on the PDA surface. However, when the metallic PDA surface is exposed to 254 nm UV light, the MNPs on PDA are oxidized and removed from PDA surface.

Herein, we demonstrated that the secondary modification of a PDA surface could be effectively accelerated under UV irradiation at $365 \mathrm{~nm}(\approx 12$ times for the thiol or amine modification and more than 550 times for metallization). The extent of UV-assisted metallization on a PDA surface was higher than that of conventional method in dark environment. Meanwhile, the MNPs affixed on a PDA surface remained a highly uniform feature, with even distribution and small size dispersion. Moreover, we showed that the MNPs deposited on a PDA surface could be dissolved by applying UV irradiation at $254 \mathrm{~nm}$, rendering the dynamic deposition and removal of MNPs on it (Figure 1). The possible mechanisms for the rapid deposition and removal of MNPs on PDA surfaces were investigated and discussed. Due to the spatial and temporal controllability of light, the metallization of PDA surface could be well controlled, allowing the construction of a grayscale patterned metallic surface. Reversible depositing and removing MNPs enabled us to recycle metals from wastewater and electric circuits. Thus, this method demonstrates rapid, controllable and reversible secondary modification of PDA coatings.

\section{Results and Discussion}

A PDA surface was prepared by a dip-coating process using glass plate as substrate. ${ }^{[1]}$ As shown in Figure 2ai, after $24 \mathrm{~h}$ deposition, the glass plate turned from being transparent to light brown, indicating the deposition of a thin PDA layer on the glass surface. Immersing this PDA-modified surface into $\mathrm{AgNO}_{3}$ solution would trigger the spontaneously $\mathrm{Ag}^{+}$reduction by PDA, to deposit Ag NPs onto the PDA surface. After immersion in $0.1 \mathrm{M} \mathrm{AgNO}_{3}$ aqueous solution for $24 \mathrm{~h}$, the surface color became slightly darker, indicating the deposition of $\mathrm{Ag}$ NPs on the PDA surface. Deposition of Ag NPs on PDA surface was confirmed by scanning electron microscope (SEM) analysis before and after metallization treatment (Figure 2aii).

We observed that by UV irradiation $\left(365 \mathrm{~nm}, 4.3 \mathrm{~mW} \mathrm{~cm}{ }^{-2}\right.$ ) on the PDA surfaces during immersion, the reduction process was significantly accelerated. As shown in Figure 2aiii, after $30 \mathrm{~min}$ UV irradiation in $0.1 \mathrm{M} \mathrm{AgNO}_{3}$ solution, the PDA-coated glass plate turned dark brown, and the generated Ag NPs increased dramatically. We counted the Ag NPs on the PDA surfaces after $\mathrm{AgNO}_{3}$ treatment (in the dark and under
UV irradiation). The conventional metallization method (24 h in the dark; Figure 2aii) generated Ag NPs with $55 \pm 8 \mu \mathrm{m}^{-2}$ density. While under UV irradiation for $30 \mathrm{~min}$ (Figure 2aiii), Ag NPs with $265 \pm 12 \mu \mathrm{m}^{-2}$ density were deposited on the PDA surface, revealing much faster reduction kinetics. On the contrary, no Ag NPs were observed on a bare glass substrate after the same treatment $\left(0.1 \mathrm{M} \mathrm{AgNO}_{3}\right.$ solution, $30 \mathrm{~min}$ UV irradiation), confirming the key role of PDA played in the $\mathrm{Ag}^{+}$ reduction process (Figure 2aiv). We conducted XPS analysis on the PDA surface after $\mathrm{Ag}^{+}$reduction $\left(0.1 \mathrm{M} \mathrm{AgNO}_{3}\right.$ solution, $30 \mathrm{~min}$ UV), and a clear Ag $3 \mathrm{~d}$ peak was apparent (Figure 2b). However, the Ag $3 \mathrm{~d}$ peak was undetectable on the pristine PDA surface, confirming the in situ formation of Ag NPs on the PDA surface under UV irradiation. A detailed analysis of the C 1s peak of Ag NP-deposited PDA and bare PDA revealed no distinct difference (Figure 2b), indicating that PDA coating's composition did not change significantly after $\mathrm{AgNO}_{3}$ and UV treatments. Similar results were also found in their Raman spectra, as shown in Figure S1 (Supporting Information).

To quantitatively investigate the acceleration effect of UV irradiation on metallization of the PDA surface, we measured the UV-vis absorbance of PDA-modified glass during the metallization process $\left(0.1 \mathrm{M} \mathrm{AgNO}_{3}\right.$ solution) along with the duration of UV irradiation. As a control, the UV-vis absorbance of the nonirradiated sample was also measured. As shown in Figure 2c, the absorbance of the PDA surface at $440 \mathrm{~nm}$ (corresponding to plasmon resonance absorption of $\mathrm{Ag}$ ) clearly increased along with UV irradiation, from 0.53 to 0.64 after $10 \mathrm{~min}$ UV irradiation, and reached 2.25 after $2 \mathrm{~h}$ irradiation. In comparison, after $24 \mathrm{~h}$ metallization treatment in the dark, the nonirradiated PDA surface still exhibited small absorbance $(0.70$ at $440 \mathrm{~nm})$, which equaled that of the sample after 10-20 min UV irradiation. We thus concluded that the extent of metallization driven by UV irradiation far exceeded that of the conventional route in dark, and the metallization had been accelerated about 140 times by UV irradiation with intensity of $4.3 \mathrm{~mW} \mathrm{~cm}^{-2}$ (unless otherwise stated, all acceleration calculations are compared with the conventional method in dark environment). Besides the UV irradiation time, we investigated the UV intensity's effect. The metallization process (in $0.1 \mathrm{M} \mathrm{AgNO}_{3}$ solution) was conducted under 1.6, 4.3, and $21.6 \mathrm{~mW} \mathrm{~cm}{ }^{-2} \mathrm{UV}$ irradiation, respectively. The absorbance (at $440 \mathrm{~nm}$ ) of the PDA surface exhibited obvious dependence 
(a) $\mathrm{i}$
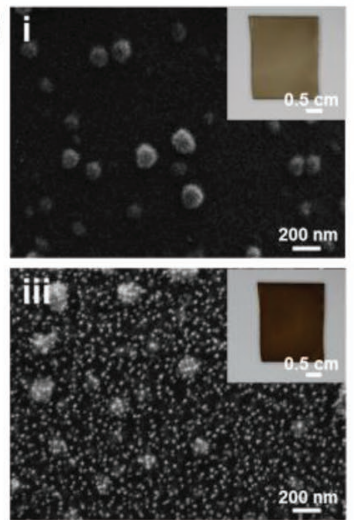

(c)

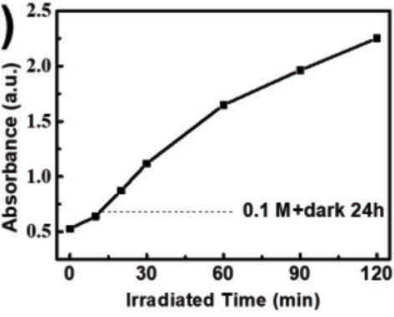

(f)

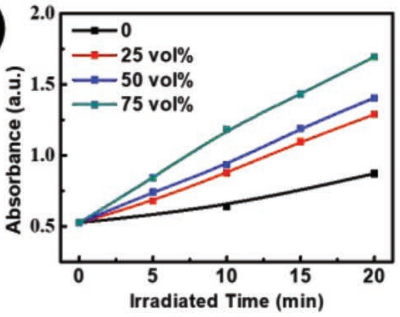

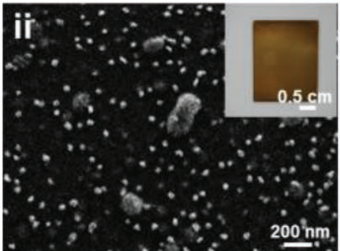

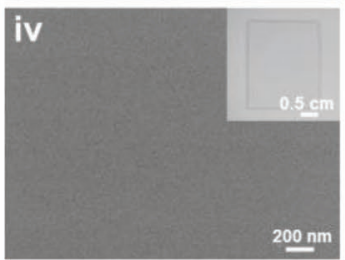

(b)
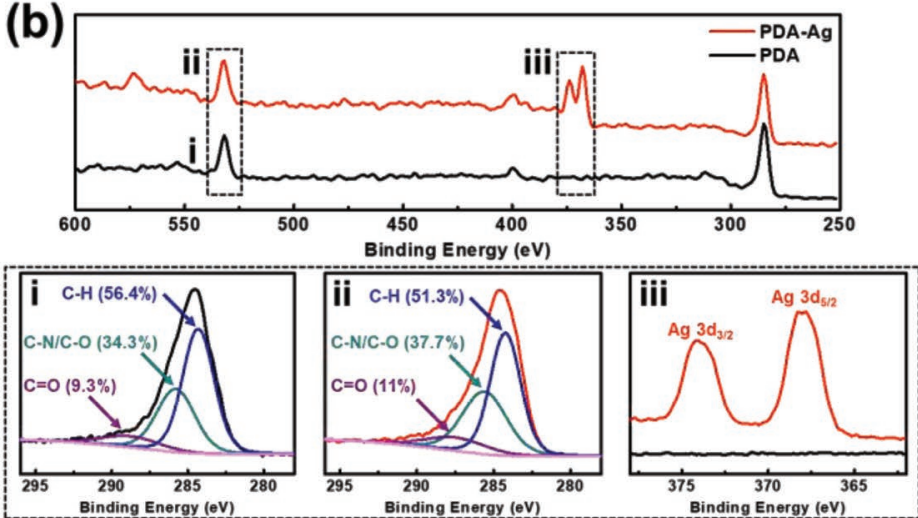

(e)

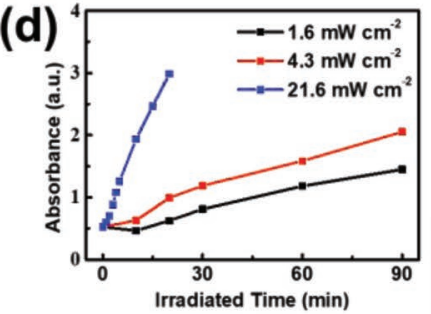

(g)

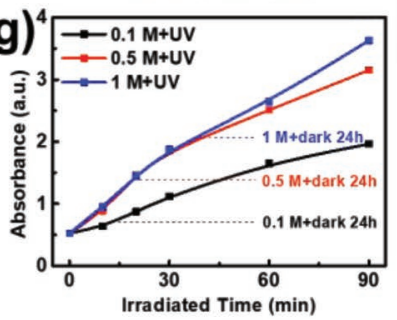

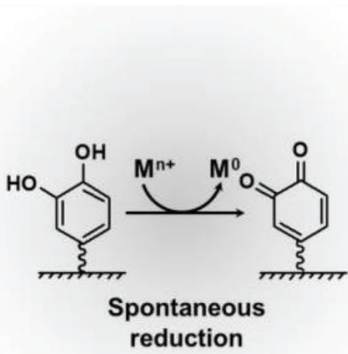

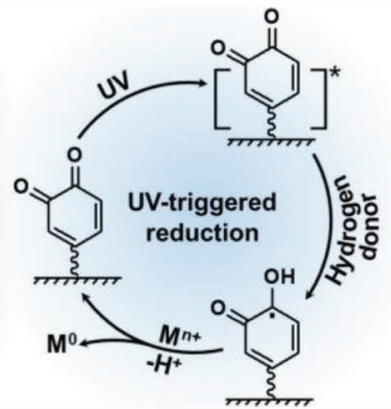

Figure 2. a) Digital photographs and SEM images of PDA-modified glass plate i), PDA-coated glass after $24 \mathrm{~h}$ immersion in AgNO 3 solution (0.1 $\mathrm{M}$, in dark) ii), PDA-coated glass after 30 min UV irradiation $\left(4.3 \mathrm{~mW} \mathrm{~cm}^{-2}\right.$ at $\left.365 \mathrm{~nm}\right)$ in $\mathrm{AgNO}_{3}$ solution (0.1 M) iii), and bare glass substrate after 30 min UV irradiation $\left(4.3 \mathrm{~mW} \mathrm{~cm}{ }^{-2}\right.$ ) in $\mathrm{AgNO}_{3}$ solution $\left.(0.1 \mathrm{~m}) \mathrm{iv}\right)$. b) XPS full spectra of a bare PDA surface and PDA-Ag composite surface obtained after UV irradiation $\left(4.3 \mathrm{~mW} \mathrm{~cm}{ }^{-2}\right.$ ) in $0.1 \mathrm{M} \mathrm{AgNO}_{3}$ solution for $30 \mathrm{~min}$. The figures inside the dotted frames are the high-resolution $\mathrm{C} 1 \mathrm{~s}$ peak of a bare PDA surface i), PDA-Ag surface ii), and the Ag $3 \mathrm{~d}$ peak of these two surfaces iii). c) The UV time-dependent UV-vis absorbance (at 440 nm) of a PDA-modified glass plate irradiated with $4.3 \mathrm{~mW} \mathrm{~cm}^{-2} \mathrm{UV}$ light in $0.1 \mathrm{M} \mathrm{AgNO}_{3}$ solution. The dotted line represents the absorbance (at $440 \mathrm{~nm}$ ) of PDA coated glass after $24 \mathrm{~h}$ immersion in $\mathrm{AgNO}_{3}$ solution $(0.1 \mathrm{M}$, in dark). d) The UV irradiation time dependent UV-vis absorbance (at 440 nm) of a PDA surface after irradiated with different dose of UV light in $0.1 \mathrm{M} \mathrm{AgNO}$ solution. e) Illustration of the mechanisms of spontaneous reduction of metal cations on a PDA surface and UV-triggered reduction of metal cations on PDA surface. $f$ ) The UV irradiation time dependent UV-vis absorbance (at $440 \mathrm{~nm}$ ) of a PDA-modified surface after irradiation with $4.3 \mathrm{~mW} \mathrm{~cm} \mathrm{mV}^{-2} \mathrm{UV}$ light in $0.1 \mathrm{~m} \mathrm{AgNO}_{3}$ solution with different ethanol concentrations. g) The UV irradiation time dependent UV-vis absorbance (at $440 \mathrm{~nm}$ ) of PDA-modified surface after irradiation with $4.3 \mathrm{~mW}^{-2} \mathrm{~cm}^{-2} \mathrm{UV}$ light in AgNO 3 solution with different concentrations. The dotted lines represent the absorbance (at $440 \mathrm{~nm}$ ) of PDA-coated glass after immersion in $0.1,0.5$, and $1 \mathrm{M} \mathrm{AgNO}_{3}$ solution (24 h, in the dark).

on the UV intensity. Higher UV intensity led to faster Ag NP deposition (Figure 2d). For the $\mathrm{Ag}^{+}$reduction under $21.6 \mathrm{~mW} \mathrm{~cm}{ }^{-2}$ UV irradiation, metallization was accelerated more than 550 times, confirming UV's acceleration effect. Interestingly, although the use of UV irradiation speeded up the reduction and Ag NP density significantly, the good uniformity and dispersity of Ag NPs were well maintained, as Figure S2 (Supporting Information) shows (the size and density of Ag NPs on these samples, as shown in Figure S3 (Supporting Information), demonstrate that both size and density of Ag NPs increased with UV irradiation time as well as UV light intensity). This observation/uniformity was different from conventional photoreduction methods based on a photocatalytic semiconductor, such as $\mathrm{TiO}_{2}$, which generates anisotropic MNPs with large size distribution and uncontrollable morphology under UV irradiation. ${ }^{[27,28]}$
The reason for this phenomenon is not clear yet, but we assume that PDA might act as a capping agent to stabilize and confine the deposited Ag NPs, in addition to reducing metal cations. . $^{[4,29,30]}$

The accelerated $\mathrm{Ag}^{+}$reduction via $\mathrm{UV}$ is clearly observable by using direct laser writing (DLW) to trigger the reaction. As Figure S4a (Supporting Information) illustrates, during the $50 \mathrm{~s}$ UV irradiation, the PDA surface immersed in $0.1 \mathrm{M} \mathrm{AgNO}_{3}$ clearly darkened at the exposed area (also shown in Video S1 in the Supporting Information), implying the in situ formation of Ag NPs. However, when the UV was off, no change in brightness was observed during the subsequent $130 \mathrm{~s}$ (Figure S4b, Supporting Information), confirming the high UV dependence of Ag NP formation in this case. On the contrary, no color change appeared on the unexposed area of the PDA surface or 
irradiated bare glass surface (Figure S4aiv,v and Video S2, Supporting Information), proving the necessity of both UV irradiation and PDA in this rapid reduction process.

Based on these observations, we propose a possible mechanism for the phototriggered reduction of metal cations on a PDA surface. As illustrated in Figure 2e, PDA is known to be composed of catechol and quinone groups. ${ }^{[31]}$ The spontaneous metal cations reduction process on a PDA surface involves the oxidation of catechol (to quinone) and the simultaneous reduction of metal cations to MNPs. ${ }^{[32]}$ However, this process is slow and catechol-consuming. Very few MNPs are obtained after a long time of spontaneous reduction. In the UV-triggered reduction, problems of low reaction efficiency and the consuming of catechol groups are overcame. Under UV irradiation, quinone is photoexcited to excited state and then abstracts a hydrogen from hydrogen donor surrounded (such as water). ${ }^{[33]}$ By this process, ketyl radicals could be generated which reduce metal cations, thereby leading to the formation of MNPs. Meanwhile, the PDA catechol group could also be oxidized to quinone by oxygen under UV irradiation, ${ }^{[19]}$ and participated in the UVtriggered reduction process via the aforementioned mechanism. In the UV-triggered reduction, the quinone of PDA contributes to reducing the metal cations, but is not consumed. Thus, the reduction of metal cations occurs continuously and the extent of metallization could vastly surpass that of spontaneous reduction. In this proposed UV-triggered reduction process, PDA may act alike a catalyst, which matches the results in $\mathrm{C} 1 \mathrm{~s}$ peak analysis and Raman test (Figure 2b; Figure S1, Supporting Information).

In the proposed mechanism, the presence of a hydrogen donor is very important since it leads to the formation of ketyl radicals, which then trigger metallization. Increasing the hydrogen-donor activity may speed up the entire reduction process. Ethanol is known to be a more efficient hydrogen donor than water. ${ }^{[34,35]}$ Therefore, to confirm our proposed mechanism, ethanol was added to the $\mathrm{AgNO}_{3}$ solution during the metallization process. As we expected, the absorbance (at $440 \mathrm{~nm}$ ) of the metallic PDA surface obtained increased faster at a higher ethanol concentration (Figure 2f). As no absorbance change was apparent on bare glass plates under the same treatment (Figure S5, Supporting Information), ethanol could not reduce $\mathrm{Ag}^{+}$in the absence of PDA in this case. When the solvent was 75 vol\% ethanol in water, the reduction speed was further accelerated about 500 times. We attribute this acceleration effect to the fact that, with a more efficient hydrogen donor (ethanol), the excited quinone group on PDA could abstract hydrogen more easily, and therefore form ketyl radicals more efficiently, leading to faster reduction of metal cations.

Higher $\mathrm{Ag}^{+}$concentrations are known to significantly accelerate the metallization process of PDA in the dark. To find out whether UV irradiation maintains the effect of accelerating $\mathrm{Ag}^{+}$reduction under a high $\mathrm{Ag}^{+}$concentration, we performed UV-triggered reduction under different $\mathrm{AgNO}_{3}$ concentrations (0.1, 0.5, $1 \mathrm{~m}$; Figure $2 \mathrm{~g})$. With an increased $\mathrm{Ag}^{+}$concentration (from 0.1 to $0.5 \mathrm{M}$ ), absorbance of the obtained substrates (at $440 \mathrm{~nm}$ ) rose dramatically, indicating that more Ag NPs were generated at higher $\mathrm{Ag}^{+}$concentrations. However, when we raised $\mathrm{Ag}^{+}$concentration further to $1 \mathrm{M}$, the $\mathrm{Ag} \mathrm{NP}$ formation became independent of $\mathrm{Ag}^{+}$concentration. This might because that nucleation and further nanoparticle growth tended to be saturated under such high $\mathrm{Ag}^{+}$concentration. Nevertheless, UV irradiation continued to exhibit a significant acceleration effect when the $\mathrm{Ag}^{+}$concentration was high, the reduction of $\mathrm{Ag}^{+}$was accelerated for more than 37 times even at $1 \mathrm{M} \mathrm{Ag}^{+}$concentration (see the dotted lines in Figure 2g).

As PDA absorbs light throughout the visible spectrum, we assumed that visible light could also trigger the formation of ketyl radicals on PDA surfaces, and hence accelerate metal cations' reduction. To confirm our hypothesis, two visible light sources (455 and $530 \mathrm{~nm}, 30 \mathrm{~mW} \mathrm{~cm}{ }^{-2}$ ) were applied on PDA surfaces (immersed in $0.1 \mathrm{M} \mathrm{AgNO}_{3}$ solution) for $30 \mathrm{~min}$, respectively. As Figure S6a (Supporting Information) shows, visible light clearly accelerated the $\mathrm{Ag}^{+}$reduction process. The light with shorter wavelength led to more Ag NPs, probably because that PDA exhibited higher absorbance of light with a shorter wavelength (Figure S6b, Supporting Information), thus ketyl radicals were excited more effectively by light with shorter wavelengths rather than longer wavelengths.

In addition to $\mathrm{Ag}^{+}$reduction, UV irradiation also displayed a significant acceleration effect to other types of secondary modification on PDA surfaces, such as $\mathrm{Au}^{3+}$ reduction, and reactions of PDA with thiols and amines. Few reports have addressed $\mathrm{Au}^{3+}$ reduction by $\mathrm{PDA},[4,36,37]$ while a long reaction time is required and poor $\mathrm{Au}$ NPs yields are commonly obtained. Our experiment confirmed this: as shown in Figure S7a (Supporting Information) after immersing PDA surface in $\mathrm{HAuCl}_{4}$ solution $\left(10 \times 10^{-3} \mathrm{M}\right)$ for $30 \mathrm{~min}$, the PDA surface color was practically unchanged, and few Au NPs were observed in the SEM image, indicating the difficulty of $\mathrm{Au}^{3+}$ spontaneous reduction by PDA. On the contrary, UV irradiation drove the fast reduction of $\mathrm{Au}^{3+}$, leading to high-density $\mathrm{Au}$ NPs on PDA surface after 30 min exposure (Figure S7b, Supporting Information). UV irradiation also revealed an acceleration effect on thiol and amine modification on PDA surfaces. As Figure S8a,b (Supporting Information) illustrates, when the PDA surface was treated with dodecanethiol (DT) solution or dodecylamine (DA) solution (in the dark) respectively, the water contact angle (WCA) on the surface was quickly increased from $33 \pm 4^{\circ}$ to $58 \pm 1^{\circ}$ in the first $5 \mathrm{~min}$, and then grew moderately for the next $55 \mathrm{~min}$ (from $58 \pm 1^{\circ}$ to $63 \pm 3^{\circ}$ ). However, under UV irradiation, the WCA on the PDA surface increased rapidly for $20 \mathrm{~min}$, attaining a great extent and then started to slowly increase in the following $40 \mathrm{~min}$. This phenomenon is probably because the Michael-addition (or Schiff base reaction) of thiol and amine on PDA quinone is a fast process, while the oxidation of catechol to quinone is slow (Figure S8c, Supporting Information). When we put the modification solution onto the PDA surface, the quinone groups on it reacted quickly with DT or DA, leading to a rapid WCA increase in the first $5 \mathrm{~min}$. The fast noncovalent interaction of DT or DA with PDA may also contribute to the WCA increase in the first $5 \mathrm{~min}$. Thus, the kinetics of modification was determined by the catechol oxidation process (Figure S8c, Supporting Information), which proceeded very slowly in the dark and was relatively fast under UV irradiation. For the thiol and amine modification on PDA surface, UV irradiation $\left(4.3 \mathrm{~mW} \mathrm{~cm}{ }^{-2}\right.$ at $\left.365 \mathrm{~nm}\right)$ accelerated the process $\approx 12$ times (compared to the modification in dark). 
Interestingly, UV irradiation not only causes the fast deposition of MNPs on a PDA surface, but also allows the removal of the MNPs deposited on the PDA surface. As shown in Figure 3a, a PDA surface with deposited Ag NPs was quite stable in ethanol, as no change was observed in its appearance and SEM image after $24 \mathrm{~h}$ immersion. However, when UV light at $254 \mathrm{~nm}$ was applied, the color of Ag NP-deposited PDA surface gradually faded and Ag NPs became smaller and finally invisible. The UV-vis spectra showed that the metallized PDA surface almost returned to the original noncoated PDA after $1 \mathrm{~h}$ UV exposure (254 nm), while the UV-vis absorption of a such surface remained constant without UV (Figure $3 \mathrm{~b}$ ). To better reveal the mechanism of the UV-triggered Ag removal on PDA-Ag composite surface, XPS analysis was performed on a Ag NP-deposited PDA surface before and after $254 \mathrm{~nm}$ UV exposure. As shown in Figure 3c, the $\mathrm{Ag} \mathrm{3d} / \mathrm{C} 1 \mathrm{~s}$ ratio dropped from 0.067 to 0.039 after $1 \mathrm{~h}$ exposure, indicating that a considerable amount of $\mathrm{Ag}$ had been removed from the PDA surface, probably via oxidative dissolution of silver. Limited by the resolution of SEM, the TEM test was also conducted to analyze the metallic PDA surface before and after UV irradiation $(254 \mathrm{~nm})$. As shown in Figure 3di,ii, before UV exposure, Ag NPs were anchored on PDA with high density and good dispersity. After $1 \mathrm{~h}$ $254 \mathrm{~nm}$ UV exposure, the total amount of Ag decreased significantly and the initial Ag NPs had been replaced by denser ultrafine Ag NPs $(<10 \mathrm{~nm})$ (see Figure 3diii,iv). This may indicate that the removal process consists of two parts: the dissolution of initial Ag NPs, and the regeneration of ultrafine Ag NPs. This Ag removal process did not occur in water, and hardly occurred in water/ethanol 1:1 (vol) solution or in ethanol (365 nm UV irradiation). The detailed mechanism of the phototriggered Ag removal on PDA is unclear and more studies are necessary to elucidate it. We believe that this is a PDA/ethanol-assisted Ag photo-oxidation process (Figure 3e). It has been reported that Ag NPs can be oxidized to $\mathrm{Ag}^{+}$by oxygen under UVB irradiation, due to the formation of reactive oxygen species (ROS) under UV. ${ }^{[38]}$ However, this process is quite slow (several days) and inefficient. In our experiment, PDA and ethanol probably accelerate this process by many folds, leading to significant volume loss of Ag NPs in $1 \mathrm{~h}$. Besides the Ag dissolution, part of the dissolved $\mathrm{Ag}^{+}$can also be reduced by PDA under UV, just like the proposed reduction process shown in Figure 2e. By this dynamic oxidationreduction process, the original Ag NPs are gradually reduced in size, while some ultrafine Ag NPs emerge (ultrasmall size may be caused by the extremely low concentration of free $\mathrm{Ag}^{+}$). Besides the removal of $\mathrm{Ag}$, phototriggered $\mathrm{Au}$ removal on PDA is also possible, as shown in Figure 3fii. The photocontrolled deposition and removal of MNPs on PDA surface will enable us to "write and erase" MNPs on PDA, which is smart and shows great potential.

Due to the spatial and temporal controllability of light, the metallization of PDA surfaces can be well controlled, enabling the generation of complex metallic patterns with specific metallization extents at specific locations-a process that is currently difficult or impossible using other methods. For example, by applying a photomask, we created high-density Ag NPs patterns on various PDA-coated substrates such as glass (Figure 4ai), polyimide (Figure 4aii), Teflon (polytetrafluoroethylene) (Figure 4aiii), and a hydrogel (Figure 4aiv). The deposition of MNPs can even be controlled to form gradients by using suitable photomasks (Figure 4b,c), which is quite difficult to be achieved by other patterning technologies such as physical vapor deposition (PVD), photolithography, and microcontact printing. The comparison of the original photomask and the obtained grayscale Ag NPs pattern showed that the details of the original image had been well preserved (Figure S9a,b, Supporting Information), indicating the great precision of this phototriggered patterning process. Furthermore, as Figure S9c (Supporting Information) shows, the density and morphology of Ag NPs were well controlled by the photomask. The capacity to remove MNPs is available for not only the MNPs obtained via a phototriggered reduction process but also for those obtained by any other methods such as PVD. As an illustration, we fabricated a conductive Au circuit on a PDA surface by PVD. We then subjected the Au circuit chip to a photoremoval process. As expected, the Au circuit was entirely removed from the substrate, but persisted on the PDA surface when no UV had been applied (Figure 4d). This is a good example by which to demonstrate the value of PDA in recycling valuable metals on electronic circuits, which is an expensive and very environmentally polluting process by standard commercial methods. ${ }^{[39]}$ A combinative utilization of UV-triggered deposition and removal of MNPs on PDA surfaces is illustrated in Figure 4e. PDA is known to be able to capture heavy metal ions from polluted wastewater. ${ }^{[40]}$ Here, UV irradiation significantly accelerated the process and enabled the reversible use of a PDA surface. We fabricated a PDA-coated textile and immersed it in $\mathrm{Ag}^{+}$wastewater. After $30 \mathrm{~min}$ at $365 \mathrm{~nm} \mathrm{UV}$ exposure, the $\mathrm{Ag}^{+}$was successfully enriched by the PDA textile and a Ag-deposited PDA textile was obtained. Following secondary $254 \mathrm{~nm}$ UV irradiation, the Ag absorbed on the PDA surface was released to solution in ionic form, resulting in a recycled PDA/textile that could be used twice.

\section{Conclusions}

In summary, we proposed a rapid, controllable, and reversible method to achieve secondary modification of PDA surfaces. We demonstrated that $365 \mathrm{~nm}$ UV irradiation accelerated the metallization process on PDA surface by more than 550 times, while the addition kinetics of thiol and amine to the PDA surface was increased $\approx 12$ times. This method significantly increased the MNP density on the obtained PDA/MNPs composite surfaces while maintaining uniformity and dispersity of the deposited MNPs. The extent of secondary modification on a PDA surface could be spatiotemporally controlled by applying customized photomasks during irradiation. Furthermore, we discovered that MNPs deposited on PDA could be removed under $254 \mathrm{~nm}$ UV irradiation, enabling a dynamic and reversible metallization process. Combining these features with the spatiotemporal controllability of UV irradiation and the substrate-independent coating property of PDA, this method reveals exciting new potential for constructing functional coatings and patterns with promising applications in various fields. 
(a)
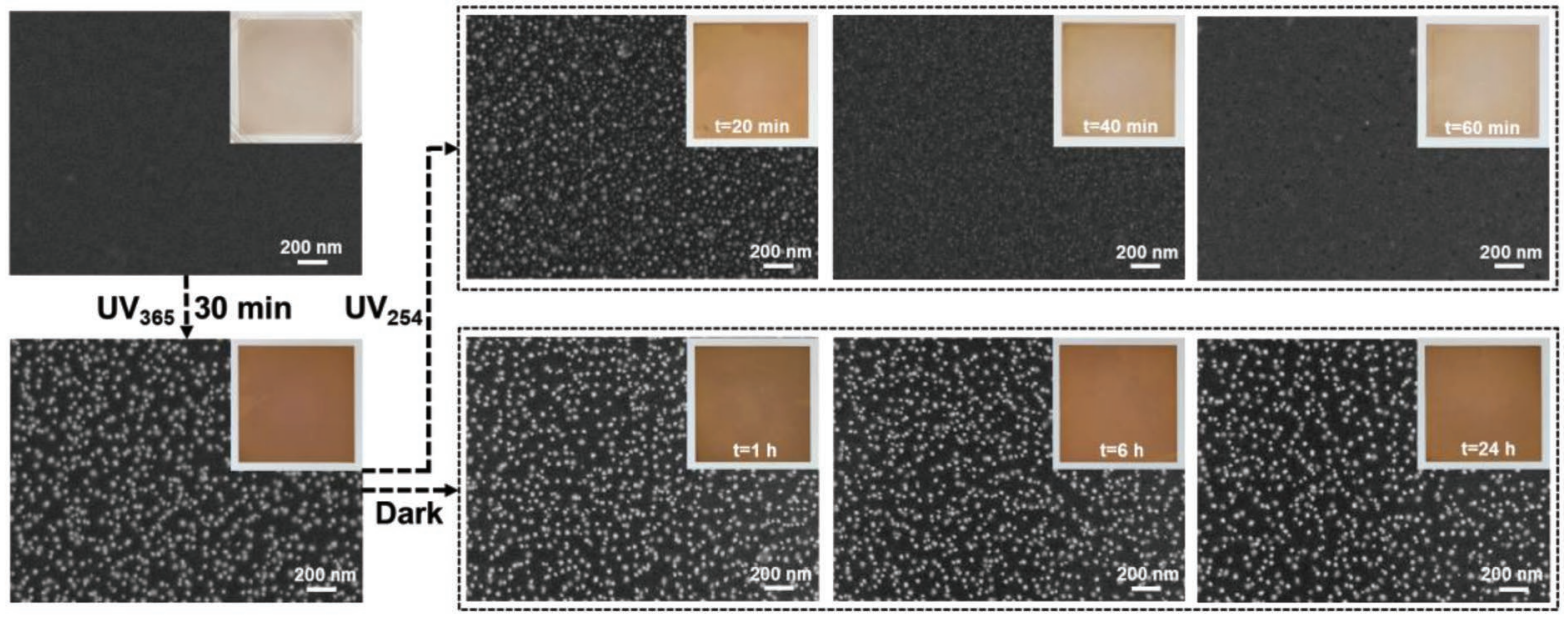

(b)

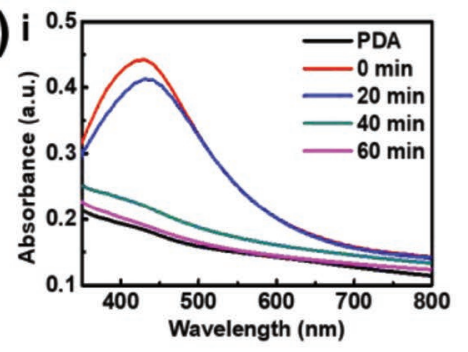

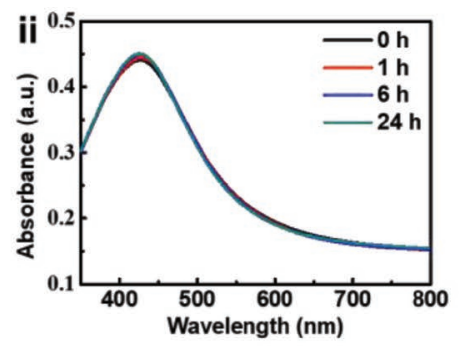

(c)



(d) $\mathrm{i}$
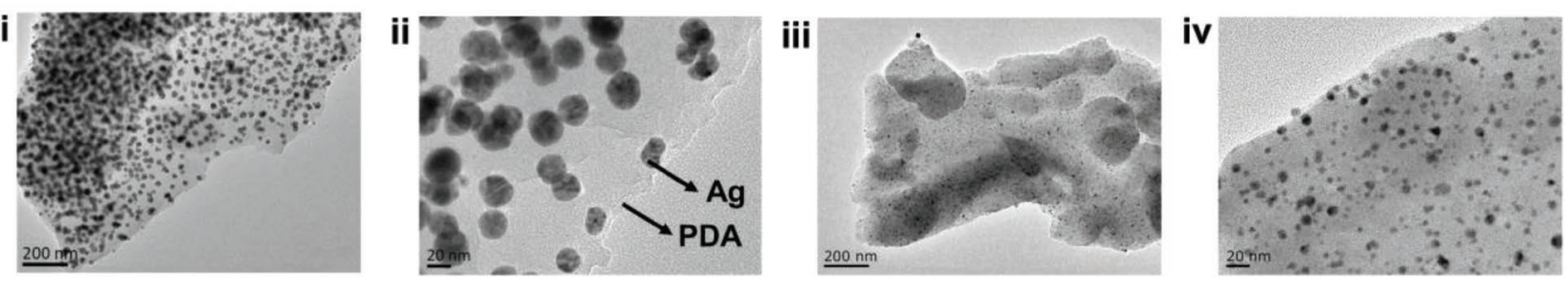

(e)

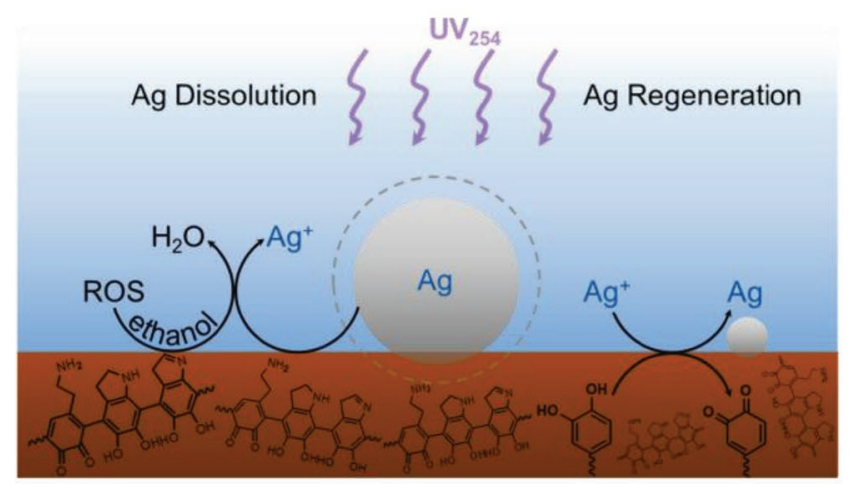

(f) $\mathrm{i}$

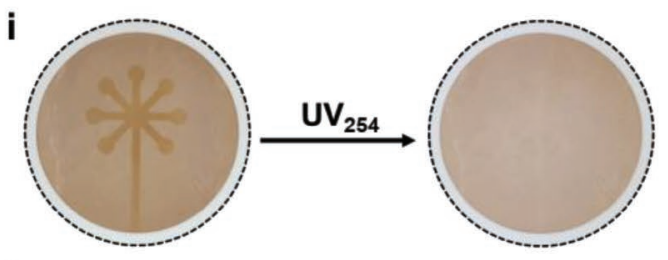

ii

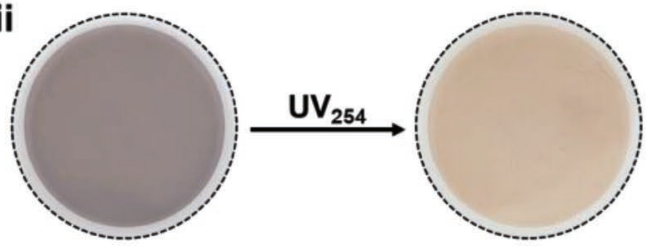

Figure 3. a) Digital photographs and SEM images of PDA-modified glass plate $(24 \times 24 \mathrm{~mm})$ after different treatments. PDA-modified glass plate was immersed in $0.1 \mathrm{M} \mathrm{AgNO}_{3}$ solution and irradiated with $365 \mathrm{~nm}$ UV light for 30 min. After that, the Ag NP-deposited PDA surface was irradiated with $254 \mathrm{~nm}$ UV light in ethanol to remove Ag NPs or directly immersed in ethanol and kept in the dark as control. The digital pictures and SEM images at different time points are recorded as shown in the dotted boxes. b) The UV-Vis spectra of samples in (a). i) The UV-vis spectra of a Ag NP-deposited PDA surface after secondary UV $(254 \mathrm{~nm})$ irradiation in ethanol. ii) The UV-vis spectra of Ag NP-deposited PDA surface after immersed in ethanol and kept in dark. c) XPS full spectra of a Ag NP-deposited PDA surface before and after $254 \mathrm{~nm}$ UV irradiation. d) TEM images of a Ag NP-deposited PDA before i,ii) and after iii,iv) $254 \mathrm{~nm}$ UV irradiation. Here, images (ii) and (iv) are the enlarged views of images (i) and (iii), respectively. e) Schematic representation of the process of photoremoving Ag NPs deposited on PDA. f) Digital photographs of patterned Ag-NPs on a PDA surface and a Au NP-deposited PDA surface (diameter of the substrates: $24 \mathrm{~mm}$ ) before and after $254 \mathrm{~nm}$ UV irradiation treatment, respectively. To facilitate the photoremoval process, potassium iodide (KI) was added as a ligand of Au to lower the potential of $\mathrm{Au}^{0} / \mathrm{Au}^{+}(+1.83 \mathrm{~V})$ and $\mathrm{Au}^{0} / \mathrm{Au}^{3+}(+1.53 \mathrm{~V})$. In that way, Au could be oxidized at much lower potential $\left(\mathrm{Au}^{3}+4 \mathrm{I}^{-}=\left[\mathrm{AuI}_{4}\right]^{-}+3 \mathrm{e}^{-},+0.56 \mathrm{~V}\right.$; $\left.\mathrm{Au}+2 \mathrm{I}^{-}=\left[\mathrm{AuI}_{2}\right]^{-}+\mathrm{e}^{-},+0.58 \mathrm{~V}\right) \cdot{ }^{[41]}$ 
(a)
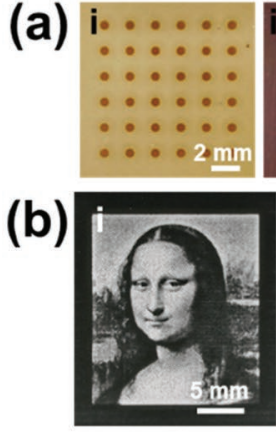

(c)

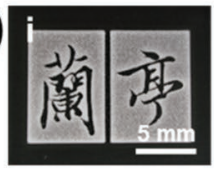

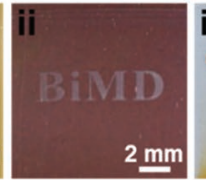
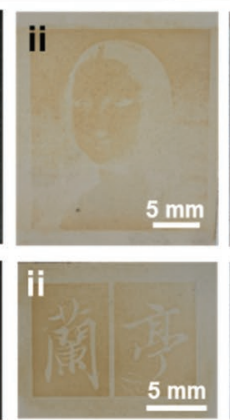
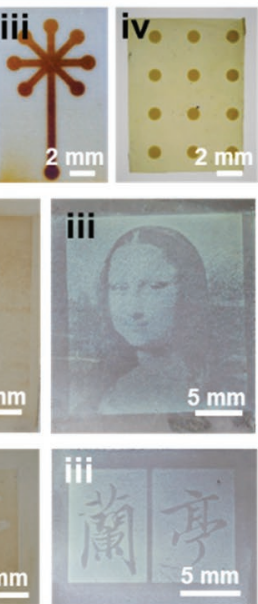

(d)
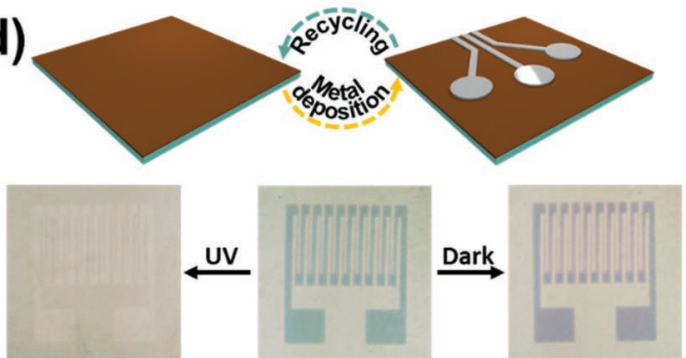

(e)

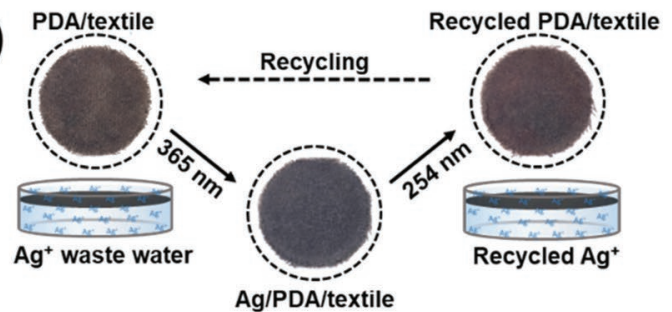

Figure 4. a-c) Various metallic patterns fabricated on various PDA-coated substrates by using corresponding photomasks. a) Ag NPs-deposited metallic patterns fabricated on PDA-modified glass plate (i), polyimide film (ii), Teflon film (iii), and hydrogel (iv) by using binary photomasks. b,c) Ag NPs-deposited metallic patterns on PDA-modified glass plates by using grayscale photomasks. The left column of b) and c) shows the grayscale photomasks fabricated by laser printer (head of "Mona Lisa," painted by Leonardo da Vinci, 1503; characters from "Lan Ting Ji Xu," written by Xizhi Wang, A.D. 353); the middle and right columns are transmission and reflection images of corresponding metallic patterns, respectively. d) A PDA surface was used to recycle electronic circuit. The upper row is the schematic diagram for recycling the electronic circuit deposited on a PDA surface. The second row shows that the PVD sputtered gold circuit on the PDA surface was successfully removed after UV irradiation (254 nm), while the pattern remained unchanged without UV irradiation. e) a PDA-coated textile (diameter $60 \mathrm{~mm}$ ) was utilized for wastewater treatment. After UV irradiation at $365 \mathrm{~nm}$, $\mathrm{Ag}^{+}$in wastewater was rapidly reduced and absorbed on PDA surface. After that, the Ag NPs absorbed on PDA were oxidized into $\mathrm{Ag}^{+}$and liberated into solution for the purpose of recycling. Thus, the recycled PDA/textile was used for the secondary wastewater disposal.

\section{Experimental Section}

Materials: dopamine hydrochloride (AR), tris(hydroxymethyl) aminomethane- $\mathrm{HCl}$ (Tris- $\mathrm{HCl}, 1.5 \mathrm{~m}, \mathrm{pH}=8.8$ ), 1-dodecanethiol (98\%), and dodecylamine $(98 \%)$ were purchased from Macklin Biochemical Co., Ltd. (Shanghai, China). Silver nitrate $\left(\mathrm{AgNO}_{3}, \mathrm{AR}\right)$, tetrachloroauric acid hydrate $\left(\mathrm{AuCl}_{3} \cdot \mathrm{HCl} \cdot 4 \mathrm{H}_{2} \mathrm{O}, \mathrm{AR}\right)$, ethanol $(\mathrm{AR})$, sodium hydroxide $(\mathrm{NaOH}$, $A R$ ), and potassium iodide (KI, AR) were purchased from Sinopharm Chemical Reagent Co., Ltd. (China). During the experiments the chemicals were weighted by a METTLER TOLEDO MS205DU electronic balance.

Fabrication of PDA Coatings: the substrate was immersed in stirring dopamine solution $\left(2 \mathrm{mg} \mathrm{mL}^{-1}, 10 \times 10^{-3} \mathrm{M}\right.$ Tris-HCl). After $24 \mathrm{~h}$, the PDA-modified substrate was obtained by rinsing it in water and drying under room temperature.

Phototriggered Reduction Process: The PDA-modified substrate was immersed in $\mathrm{AgNO}_{3}$ aqueous solution or $\mathrm{HAuCl}_{4}$ solution, and irradiated with required light. After irradiation, the obtained PDA-Ag or PDA$\mathrm{Au}$ composite surfaces were washed in water and dried under room temperature. To fabricate an adequate metallic pattern, the customized photomask was positioned between a sample and light source, then the reduction of metal cations happened in the exposed area.

Photoremoval Process: To remove the Ag NPs on PDA surface, a Ag NP-deposited PDA surface was immersed in ethanol and irradiated with $254 \mathrm{UV}$ light $\left(6 \mathrm{~mW} \mathrm{~cm}{ }^{-2}\right)$. Au NPs were removed following the aforementioned steps in a $\mathrm{KI}$ solution $(0.5 \mathrm{M}$, solvent: 50 vol\% ethanol in water).

Thiol and Amine Modification: A PDA-coated glass was immersed in $4 \mathrm{~mL} 10 \mathrm{mg} \mathrm{mL}^{-1}$ dodecanethiol or decylamine solution (ethanol as solvent) and then exposed to $4.3 \mathrm{~mW} \mathrm{~cm} \mathrm{~cm}^{-2}$ UV light $(365 \mathrm{~nm})$. After irradiation, the obtained surface was washed in ethanol and dried under room temperature.

Characterizations: The UV-vis spectra were measured by using a UV-vis-NIR spectrophotometer (UV-6100, Mapada Instruments, Shanghai, China). SEM tests were performed using a field emission scanning electron microscope (Zeiss Ultra Plus, Carl Zeiss, Germany).
TEM tests were conducted by using a transmission electron microscope (JEM2100EX, JEOL Ltd., Japan). X-ray photoelectron spectroscopy analysis was performed on a scanning XPS microprobe instrument (PHI5000 VersaProbe, ULVAC-PHI, Japan). Direct laser writing was conducted with a 3D printing system (Photonic Professional GT, Nanoscribe $\mathrm{GmbH}$ ). The Raman spectra were obtained by Raman spectrometer (Invia microRaman, Renishaw Inc.). The diameter distribution of Ag NPs on PDA surfaces was measured by randomly selecting $100 \mathrm{Ag}$ particles and calculated with Image) software. Water-contact angles were measured with a contact angle measuring instrument (JC2000D, POWEREACH, Shanghai).

\section{Supporting Information}

Supporting Information is available from the Wiley Online Library or from the author.

\section{Acknowledgements}

This work was supported by National Key R\&D Program of China (No. 2017YFA0700500), the National Science Foundation of China (Nos. 21327902 and 51628102), the Fundamental Research Funding from Jiangsu Province (No. BK20170662), China Postdoctoral Science Foundation funded project (Nos. 2018T110428 and 2017M621597), and the Fundamental Research Funds for the Central Universities (No. 2242018R20011)

\section{Conflict of Interest}

The authors declare no conflict of interest. 


\section{Keywords}

patterning, polydopamine, secondary modification, surface metallization, UV trigger

[1] H. Lee, S. M. Dellatore, W. M. Miller, P. B. Messersmith, Science 2007, 318, 426.

[2] J. H. Ryu, P. B. Messersmith, H. Lee, ACS Appl. Mater. Interfaces 2018, 10, 7523.

[3] Z. Wang, Y. Duan, Y. Duan, J. Controlled Release 2018, 290, 56.

[4] S. Du, Y. Luo, Z. Liao, W. Zhang, X. Li, T. Liang, F. Zuo, K. Ding, J. Colloid Interface Sci. 2018, 523, 27.

[5] L. Zhang, J. Wu, Y. Wang, Y. Long, N. Zhao, J. Xu, J. Am. Chem. Soc. 2012, 134, 9879.

[6] K. M. Beckwith, P. Sikorski, Biofabrication 2013, 5, 045009.

[7] J. Chen, M. L. Mei, Q. L. Li, C. H. Chu, RSC Adv. 2016, 6, 104025.

[8] S. Kim, L. K. Jang, M. Jang, S. Lee, J. G. Hardy, J. Y. Lee, ACS Appl. Mater. Interfaces 2018, 10, 33032.

[9] H. Coskun, A. Aljabour, L. Uiberlacker, M. Strobel, S. Hild, C. Cobet, D. Farka, P. Stadler, N. S. Sariciftci, Thin Solid Films 2018, 645, 320.

[10] Y. Feng, Y. Zheng, Z. U. Rahman, D. Wang, F. Zhou, W. Liu, J. Mater. Chem. A 2016, 4, 18022.

[11] F. Zhang, H. Zhang, R. Chen, Q. Liu, J. Liu, C. Wang, Z. Sun, J. Wang, J. Colloid Interface Sci. 2019, 534, 172

[12] C. Zhang, Y. Ou, W. Lei, L. Wan, J. Ji, Z. Xu, Angew. Chem., Int. Ed. 2016, 55, 3054.

[13] Q. Liu, B. Huang, A. Huang, J. Mater. Chem. A 2013, 1, 11970.

[14] Y. Liu, Y. Sui, C. Liu, C. Liu, M. Wu, B. Li, Y. Li, Carbohydr. Polym. 2018, 188, 27.

[15] C. Dhand, M. Venkatesh, V. A. Barathi, S. Harini, S. Bairagi, E. Goh Tze Leng, N. Muruganandham, K. Z. W. Low, M. H. U. T. Fazil, X. J. Loh, D. K. Srinivasan, S. P. Liu, R. W. Beuerman, N. K. Verma, S. Ramakrishna, R. Lakshminarayanan, Biomaterials 2017, 138, 153.

[16] L. Zhang, H. Su, J. Cai, D. Cheng, Y. Ma, J. Zhang, C. Zhou, S. Liu, H. Shi, Y. Zhang, C. Zhang, ACS Nano 2016, 10, 10404.

[17] D. Y. Zhang, Y. Zheng, H. Zhang, J. H. Sun, C. P. Tan, L. He, W. Zhang, L. N. Ji, Z. W. Mao, Adv. Sci. 2018, 5, 1800581.
[18] V. Trouillet, A. Welle, P. B. Messersmith, P. A. Levkin, ACS Appl. Mater. Interfaces 2018, 10, 39268.

[19] X. Du, L. Li, J. Li, C. Yang, N. Frenkel, A. Welle, S. Heissler, A. Nefedov, M. Grunze, P. A. Levkin, Adv. Mater. 2014, 26, 8029.

[20] M. Lee, S. H. Lee, I. K. Oh, H. Lee, Small 2017, 13, 1600443.

[21] F. Behboodi-Sadabad, H. Zhang, V. Trouillet, A. Welle, N. Plumeré, P. A. Levkin, Adv. Funct. Mater. 2017, 27, 1700127.

[22] Q. Wei, F. Zhang, J. Li, B. Li, C. Zhao, Polym. Chem. 2010, 1, 1430.

[23] S. Ryu, Y. Lee, J. W. Hwang, S. Hong, C. Kim, T. G. Park, H. Lee, S. H. Hong, Adv. Mater. 2011, 23, 1971.

[24] K. Kang, S. Lee, R. Kim, I. S. Choi, Y. Nam, Angew. Chem., Int. Ed. 2012, 51, 13101

[25] M. Guardingo, M. J. Esplandiu, D. Ruiz-Molina, Chem. Commun. 2014, 50, 12548.

[26] X. Du, L. Li, F. Sadabad, A. Welle, J. Li, S. Heissler, H. Zhang, N. Plumere, P. A. Levkin, Polym. Chem. 2017, 8, 2145.

[27] Y. Zeng, X. Du, B. Gao, B. Liu, Z. Xie, Z. Gu, ACS Appl. Mater. Interfaces 2018, 10, 4222.

[28] E. Stathatos, P. Lianos, P. Falaras, A. Siokou, Langmuir 2000, 16, 2398.

[29] Y. Xie, B. Yan, H. Xu, J. Chen, Q. Liu, Y. Deng, H. Zeng, ACS Appl. Mater. Interfaces 2014, 6, 8845.

[30] X. Xu, Q. Zheng, G. Bai, L. Song, Y. Yao, X. Cao, S. Liu, C. Yao, Electrochim. Acta 2017, 242, 56.

[31] Y. Liu, K. Ai, L. Lu, Chem. Rev. 2014, 114, 5057

[32] A. E. Wendlandt, S. S. Stahl, Angew. Chem., Int. Ed. 2015, 54, 14638.

[33] M. Sakamoto, M. Fujistuka, T. Majima, J. Photochem. Photobiol., C 2009, 10, 33

[34] T. Miyagi, M. Kamei, T. Mitsuhashi, T. Ishigaki, A. Yamazaki, Chem. Phys. Lett. 2004, 390, 399.

[35] K. Matsubara, T. Tatsuma, Adv. Mater. 2007, 19, 2802.

[36] J. Han, Y. Liu, R. Guo, Adv. Funct. Mater. 2009, 19, 1112.

[37] M. Zhang, X. He, L. Chen, Y. Zhang, J. Mater. Chem. 2010, 20, 10696.

[38] A. M. Mittelman, J. D. Fortner, K. D. Pennell, Environ. Sci.: Nano 2015, 2, 683

[39] H. M. Veit, T. R. Diehl, A. P. Salami, J. S. Rodrigues, A. M. Bernardes, J. A. S. Tenorio, Waste Manage. 2005, 25, 67.

[40] Y. Li, R. Zhao, S. Chao, B. Sun, C. Wang, X. Li, Chem. Eng. J. 2018, 344, 277.

[41] Y. Konishi, I. Tanabe, T. Tatsuma, Chem. Commun. 2013, 49, 606. 
Karlsruher Institut für Technologie

\section{Repository KITopen}

Dies ist ein Postprint/begutachtetes Manuskript.

Empfohlene Zitierung:

Zeng, Y.; Du, X.; Hou, W.; Liu, X.; Zhu, C.; Gao, B.; Sun, L.; Li, Q.; Liao, J.; Levkin, P. A.; Gu, $Z$.

UV-Triggered Polydopamine Secondary Modification: Fast Deposition and Removal of Metal Nanoparticles.

2019. Advanced functional materials, 29.

doi: $10.554 / I R / 1000096038$

Zitierung der Originalveröffentlichung:

Zeng, Y.; Du, X.; Hou, W.; Liu, X.; Zhu, C.; Gao, B.; Sun, L.; Li, Q.; Liao, J.; Levkin, P. A.; Gu, $Z$.

UV-Triggered Polydopamine Secondary Modification: Fast Deposition and Removal of Metal Nanoparticles.

2019. Advanced functional materials, 29 (34), Article: 1901875.

doi:10.1002/adfm.201901875 